
\title{
25 Research Soure \\ Safety and efficacy of current sclera fixation methods for intraocular lenses
}

\section{Daniel Rudolf Muth ( $\nabla$ dr-muth@t-online.de)}

Department of Ophthalmology, University Hospital, LMU Munich

\section{Armin Wolf}

University of Ulm

Thomas C. Kreutzer

Department of Ophthalmology, University Hospital, LMU Munich

Mehdi Shajari

Department of Ophthalmology, University Hospital, LMU Munich

\section{Efstathios Vounotrypidis}

University of Ulm

Siegfried G. Priglinger

Department of Ophthalmology, University Hospital, LMU Munich

\section{Wolfgang J. Mayer}

Department of Ophthalmology, University Hospital, LMU Munich

\section{Research Article}

Keywords: intraocular lens, secondary IOL, IOL exchange, pseudophakia, scleral fixation

Posted Date: May 5th, 2021

DOl: https://doi.org/10.21203/rs.3.rs-477765/v1

License: (c) (i) This work is licensed under a Creative Commons Attribution 4.0 International License. Read Full License 


\section{Abstract \\ Purpose}

Evaluation of currently three most-common intraocular lens (IOL) sclera fixation techniques (1) Prolene ${ }^{\circledR}$ suture with Hoffman sclera pocket (2) GoreTex ${ }^{\circledR}$ suture four-point technique (3) sutureless flanged intrascleral IOL fixation double-needle ("Yamane") technique.

\section{Study design}

: Retrospective, clinical case series (chart review) conducted at the Department of Ophthalmology of the Ludwig-Maximilians-University, Munich, Germany.

\section{Methods}

Fifty-seven eyes of 53 patients were included. Best-corrected visual acuity (BCVA), manifest refraction (OR), corneal tomography, biometry, central macular thickness (CMT), intraocular pressure (IOP) were recorded and compared before and at least 20 days after IOL sclera fixation surgery.

\section{Results}

GoreTex ${ }^{\circledR}$ suture was done in 15 , Prolene ${ }^{\circledR}$ in 20 , Yamane technique in 22 eyes. The GoreTex ${ }^{\circledR}$ group showed 2, Prolene ${ }^{\circledR} 3$, Yamane 1 eye that needed refixation due to visual impairment. Analysis between preand post-surgery revealed a statistically significant difference in the total BCVA $(p=0.002)$ and the Yamane subgroup BCVA ( $p=0.047)$. There was no statistical difference between pre- and post-surgery IOP, CMT and CCT. No retinal complications were found. Surgery time with Yamane technique was about 1.5 times faster than GoreTex ${ }^{\circledR}$ and Prolene ${ }^{\circledR}$. The surgery times of the subgroups differed statistical significantly.

Regarding safety aspects of the three techniques no statistically significant difference pre- and post-surgery existed.

\section{Conclusion}

A visual rehabilitation to the status pre-surgery seems to be possible with a considerable amount of revision rates. All three analyzed techniques are a successful option in secondary IOL implantation in terms of efficacy and safety. The Yamane technique seems to be the least time-consuming surgical procedure.

\section{Introduction}

We are currently seeing an increasing number of revisions of implanted intraocular lenses (IOLs). This is mainly due to the current demographic development. As life expectancy increases pseudophakic patients get older (pseudophakic ageing). Moreover, the total number of pseudophakic patients increases 
(pseudophakic shift). Hence, ophthalmologists are confronted with an increasing number of long-term complications. Those complications often result in the need for IOL refixation or IOL exchange. Reasons for IOL exchange can be due to the IOL itself such as, IOL opacification or IOL glistening, uveitis-glaucomahyphema (UGH) syndrome with iris chafing, predominantly with 1-piece sulcus-implanted IOLs ${ }^{1,2}$ with an incidence of approximately $1 \%$ per year. ${ }^{3}$ Furthermore, contact of an anterior chamber IOL with the corneal endothelium can result in IOL exchange. Another main cause for IOL revisions is IOL dislocation, be it primarily as estimated by Pueringer et al. ${ }^{4}$ with an incidence of $1.7 \%$ over 25 pseudophakic years or be it due to genetical predisposition with zonula fiber weakness such as in pseudoexfoliation syndrome (PEX) with an prevalence of 1:20 ${ }^{5}$ increasing with age, Marfan's syndrome with a prevalence of 1:10,000 and autosomaldominant inheritance pattern ${ }^{6}$, Ehlers-Danlos' syndrome with a prevalence of 1:10,000 mostly autosomaldominant ${ }^{7}$, Weill-Marchesani syndrome with a prevalence of 1:100,000 autosomal-dominant as well as autosomal-recessive ${ }^{8}$, homocystinuria with a highly variable prevalence, autosomal-recessive ${ }^{9,10}$ or high myopia ${ }^{11}$. In addition, secondary causes as trauma or previous (complicated) ophthalmologic surgeries can result in IOL dislocations. One solution to IOL complications can be IOL sclera fixation, either re-fixation of the existing IOL or sclera fixation of a secondary IOL. Especially in cases with additional iris abnormalities sclera fixation often is the only option for visual rehabilitation for the patient.

As known by literature, currently three IOL sclera fixation techniques are most commonly applied: (1) Prolene ${ }^{\circledR}$ suture (with Hoffman sclera pocket ${ }^{12}$ ), (2) GoreTex ${ }^{\circledR}$ suture, (3) sutureless flanged intrascleral IOL fixation double-needle technique ("Yamane technique") described by Yamane et al. ${ }^{13}$. As no data on a direct comparison of these three techniques existed, we conducted this study to structurally analyze safety and efficacy parameters of IOL sclera fixation with GoreTex ${ }^{\circledR}$ suture, Prolene ${ }^{\circledR}$ suture and sutureless with flanged IOL haptics.

\section{Methods}

\section{Patients}

This single-center, retrospective, observational case series was performed at the eye hospital of the LudwigMaximilians-University (LMU) Munich, Germany. Approval was obtained from the institutional review board (IRB) of the Department of Ophthalmology, Ludwig-Maximilians-University (LMU) Munich, Germany. This study adheres to the tenets of Helsinki. All patients with IOL sclera fixation were included. They were identified by filtering our patient database (SAP Deutschland SE \& Co. KG, Walldorf, Germany) by surgical procedure codes (DRG code) and material codes used for documentation of IOL sclera fixation between January 2014 and September 2019. Informed consent was obtained by all subjects.

Best-corrected visual acuity (BCVA), manifest refraction (OR), corneal tomography with central corneal thickness (CCT) (Oculus Pentacam ${ }^{\circledR}$ and Zeiss IOL Master $700^{\circledR}$ ), biometry (Zeiss IOL Master $700^{\circledR}$ ), central macular thickness (CMT) in optical coherence tomography (OCT) (Heidelberg Engineering Spectralis ${ }^{\circledR}$ ), intraocular pressure (IOP), IOL type and power were recorded before and at least 20 days after IOL sclera fixation surgery (long-term evaluation). 


\section{Surgical technique}

The three different surgical techniques were performed by five different surgeons. In each case the surgeon decided which technique was most suitable for the patient. This depended on the lens status (aphakia, single-piece, three-piece IOL) and on personal preference and experience of the surgeon. No matter which sclera fixation technique was chosen a 25-gauge pars-plana vitrectomy was performed beforehand in order to have stable intraocular conditions. Integrity of the iris was checked. No manipulation of the retina was necessary as the retina was attached in all cases.

\section{GoreTex $^{\circledR}$ suture sclera four-point fixation}

Four 25-gauge sclerostomies were made, each two millimeters away from the limbus and six millimeters from each other at the temporal and the nasal side after marking the horizontal axis. The four GoreTex ${ }^{\circledR}$ sutures were preloaded through the four islets in the haptics of the Bausch and Lomb (B\&L) Akreos Adapt $\mathrm{AO}^{\circledR} \mathrm{IOL}$. Then the IOL was implanted intraocular retropupillary with an IOL shooter (Medicel Viscoject Bio ${ }^{\circledR}$ $2.2 \mathrm{~mm}$ ). The pre-loaded sutures were grabbed with a 25-gauge Alcon MAXGRIP ${ }^{\circledR}$ forceps through the sclerotomies, externalized and knotted above the sclera. The knot was buried in the sclera. Finally, the conjunctiva was adapted and sutured at the limbal side.

\section{Prolene $^{\circledR}$ suture sclera fixation}

Lewis et al. ${ }^{14}$ first described the Prolene suture technique in combination with a sclera pocket that was later modified by Hoffman et al. in 2006. ${ }^{12,15}$ This "Hoffman technique" was used for the Prolene ${ }^{\circledR}$-sutured IOLs in this study. Two Hoffman sclera pockets were created, one at the temporal side, one at the nasal side. A polypropylene Prolene ${ }^{\circledR} 10-0$ or 9-0 suture was fixed to each of the IOL haptics via a hedge knot. Then the IOL was implanted intraocular with the IOL shooter. The ends of the suture with the long straight needle of the Prolene ${ }^{\circledR}$ suture is guided through the anterior chamber with a 27-gauge needle through the Hoffman sclera pocket. The suture is knotted, and the knot is buried within the sclera pocket.

\section{Sutureless sclera fixation with flanged IOL haptic ends ("Yamane technique")}

Scleral marks are made with a 27-gauge needle two millimeters from the limbus. At the temporal side the mark is made 2 millimeters above then horizontal axis, at the nasal side 2 millimeters below the horizontal axis. The haptics of the three-piece IOLs are then externalized at the marked sites. The external ends of the haptics are cauterized after IOL centration and thus thickened by building a flange. No suturing is needed.

\section{Statistical analysis}

All reviewed data, including clinical data of patients, such as ophthalmic conditions, central macular thickness, central corneal thickness, IOP, details of the IOL sclera fixation technique, refractive data, IOL types were categorized using Microsoft Excel (Microsoft Corporation, Redmond, WA, USA). Statistical analysis was performed using IBM SPSS (version 22 for MacOS, IBM Corporation, Armonk, NY, USA). Homogeneity of the three non-normally distributed subgroups regarding demographic and refractive characteristics age, 
spherical equivalent (SE), axial eye ball length (AL), anterior chamber depth (ACD), flattest corneal radius of anterior surface (K1) and steepest corneal radius of anterior surface (K2) was tested using Kruskall-Wallis test with an asymptotic significance ( $\mathrm{p}$ ) at $\alpha=0,05$. Explorative-descriptive analysis, test on normal distribution (Kolmogorov-Smirnov test) and Wilcoxon signed rank test ( $z$ ) with 2-tailed significance $(p)$ based on alpha $=0.05$ and critical value $=1,96$ were done. For statistically significant $z$-values Person's effect size (r) was calculated using this formula16: see formula 1 in the supplementary files section.

In order to judge Pearson's effect size it was classified according to Cohen ${ }^{17}$ :

$0.1<=r<0.3$ : weak effect size; $0.3<=r<0.5$ : medium effect size; $r>0.5$ : strong effect size

Due to the retrospective design of this study the safety parameters central macular thickness (CMT), central corneal thickness (CCT) and intraocular pressure (IOP) were primarily analyzed in a descriptive manner. However, we calculated the post-hoc statistical power of the Wilcoxon signed rank test of the best-corrected visual acuity of the total cohort using the $G *$ Power software (G*Power version 3.1 for MacOS). ${ }^{18-20}$ Statistical power was defined as $1-\beta$ error. A statistical $\beta$ error is defined as wrongful acceptance of the null hypothesis although the alternative hypothesis is true.

\section{Results}

Seventy-two eyes of 66 patients having had IOL sclera fixation were reviewed. Fifty-seven eyes $(n=57)$ of 53 patients with a complete dataset including follow-up were enrolled in this study. Patient demographics are listed in Table 1. Reasons for IOL sclera fixation were trauma $(8 ; 14,5 \%)$, previous (complicated) ophthalmic surgery $(12 ; 21,8 \%)$, abnormal zonula fibers (i.e. PEX, Marfan's) $(14 ; 25,5 \%)$, congenital aniridia $(3 ; 5,5 \%)$, uveitis $(2 ; 3,6 \%)$, pigment dispersion glaucoma $(1 ; 1,8 \%)$, aphakia (i.e. after congenital cataract extraction) (8; $14,5 \%)$, re-fixation after sclera fixation $(2 ; 3,6 \%)$, others $(5 ; 9,1 \%)$. Those eyes were provided with IOL sclera fixation as IOL refixation $(25 ; 45.5 \%)$, IOL exchange $(11 ; 20.0 \%)$, secondary IOL implantation $(16 ; 29.1 \%)$, primary IOL implantation ( $3 ; 5.5 \%)$. The mean follow-up time, the reported intraoperative complications and the design for each IOL are listed in Table 2.

The included participants were divided into three subgroups according to their sclera fixation method: 1) GoreTex $\left.{ }^{\circledR}\left(n_{G}=14\right), 2\right)$ Prolene $\left.{ }^{\circledR}\left(n_{P}=19\right), 3\right)$ sutureless flanged $\left(n_{Y}=22\right)$. The three subgroups did not show statistically significant differences concerning age and refractive data ( $S E, A L, A C D, K 1, K 2)$ (Table 1). This was tested using a Kruskall-Wallis test as the data was not normally distributed. 


\begin{tabular}{|c|c|c|c|c|}
\hline $\begin{array}{l}\text { Table } 1 \\
\text { Demographics }\end{array}$ & Total patients & GoreTex $^{\circledR}$ & Prolene $e^{\circledR}$ & $\begin{array}{l}\text { Sutureless } \\
\text { flanged } \\
\text { ("Yamane") }\end{array}$ \\
\hline $\begin{array}{l}\text { No. of } \\
\text { reviewed eyes } \\
\text { (patients) }\end{array}$ & $72(66)$ & $16(14)$ & $28(27)$ & $25(28)$ \\
\hline $\begin{array}{l}\text { No. of eyes } \\
\text { (patients) } \\
\text { included } \\
\text { (complete } \\
\text { dataset) }\end{array}$ & $57(53)$ & $15(13)$ & $20(19)$ & $22(21)$ \\
\hline $\begin{array}{l}\text { Age [years] } \\
\text { arithmetic } \\
\text { mean } \pm \text { SD } \\
{[95 \% \mathrm{Cl}]}\end{array}$ & $\begin{array}{l}63.8 \pm 19.4 \\
{[58.7 ; 69.0]}\end{array}$ & $\begin{array}{l}58.7 \pm 20.4 \\
{[47.4 ; 69.9]}\end{array}$ & $\begin{array}{l}62.8 \pm 22.4 \\
{[52.3-73.2]}\end{array}$ & $\begin{array}{l}68.4 \pm 15.4 \\
{[61.5-75.2]}\end{array}$ \\
\hline $\begin{array}{l}\text { Sex ratio of } \\
\text { eyes } \\
\text { (male/female) }\end{array}$ & $\begin{array}{l}37(65 \%) / 20 \\
(35 \%)\end{array}$ & $12 / 3$ & $14 / 6$ & $11 / 11$ \\
\hline $\begin{array}{l}\text { SE pre [D] } \\
\text { arithmetic } \\
\text { mean } \pm \text { SD } \\
{[95 \% \mathrm{Cl}]}\end{array}$ & $\begin{array}{l}4.07 \pm 7.49 \\
{[1.74-6.41]}\end{array}$ & $\begin{array}{l}6.17 \pm 7.76 \\
{[0.96-} \\
11.38]\end{array}$ & $\begin{array}{l}2.02 \pm 7.88 \\
{[-2.53-6.57]}\end{array}$ & $\begin{array}{l}4.41 \pm 6.98 \\
{[0.82-8.00]}\end{array}$ \\
\hline $\begin{array}{l}\text { SE post [D] } \\
\text { arithmetic } \\
\text { mean } \pm \text { SD } \\
{[95 \% \mathrm{Cl}]}\end{array}$ & $\begin{array}{l}-1.23 \pm 2.05 \\
{[-1.86--0.60]}\end{array}$ & $\begin{array}{l}-1.74 \pm 1.33 \\
{[-2.58-} \\
-0.90]\end{array}$ & $\begin{array}{l}-1.31 \pm 2.43 \\
{[-2.77-0.16]}\end{array}$ & $\begin{array}{l}-0.94 \pm 2.19 \\
{[-2.03-0.14]}\end{array}$ \\
\hline $\begin{array}{l}\text { AL pre }[\mathrm{mm}] \\
\text { arithmetic } \\
\text { mean } \pm \text { SD } \\
{[95 \% \mathrm{Cl}]}\end{array}$ & $\begin{array}{l}24.30 \pm 1.61 \\
{[23.87-24.74]}\end{array}$ & $\begin{array}{l}23.85 \pm 1.91 \\
{[22.80-} \\
24.91]\end{array}$ & $\begin{array}{l}24.94 \pm 1.60 \\
{[24.15-} \\
25.73]\end{array}$ & $\begin{array}{l}24.09 \pm 1.29 \\
{[23.52-} \\
24.66]\end{array}$ \\
\hline $\begin{array}{l}\text { ACD pre }[\mathrm{mm}] \\
\text { arithmetic } \\
\text { mean } \pm \\
\text { SD }[95 \% \mathrm{Cl}]\end{array}$ & $\begin{array}{l}3.82 \pm 1.11 \\
{[3.50-4.14]}\end{array}$ & $\begin{array}{l}3.67 \pm 1.37 \\
{[2.84-4.49]}\end{array}$ & $\begin{array}{l}4.01 \pm 0 .-96 \\
{[3.50-4.52]}\end{array}$ & $\begin{array}{l}3.76 \pm 1.08 \\
{[3.25-4.26]}\end{array}$ \\
\hline $\begin{array}{l}\text { TK1 [D] } \\
\text { arithmetic } \\
\text { mean } \pm \text { SD } \\
{[95 \% \mathrm{Cl}]}\end{array}$ & $\begin{array}{l}42.53 \pm 3.65 \\
{[41.51-43.54]}\end{array}$ & $\begin{array}{l}41.89 \pm 2.31 \\
{[40.61-} \\
43.17]\end{array}$ & $\begin{array}{l}41.68 \pm 2.63 \\
{[40.28-} \\
43.08]\end{array}$ & $\begin{array}{l}43.63 \pm 4.78 \\
{[41.45-} \\
45.81]\end{array}$ \\
\hline $\begin{array}{l}\text { TK2 [D] } \\
\text { arithmetic } \\
\text { mean } \pm \text { SD } \\
{[95 \% \mathrm{Cl}]}\end{array}$ & $\begin{array}{l}44.54 \pm 4.93 \\
{[43.16-45.91]}\end{array}$ & $\begin{array}{l}43.80 \pm 1.79 \\
{[42.81-} \\
44.79]\end{array}$ & $\begin{array}{l}43.99 \pm 2.32 \\
{[42.76-} \\
45.23]\end{array}$ & $\begin{array}{l}45.48 \pm 7.36 \\
{[42.13-} \\
4 \underline{8} .83]\end{array}$ \\
\hline $\begin{array}{l}\text { Reasons for } \\
\text { IOL sclera } \\
\text { fixation }\end{array}$ & $\begin{array}{l}8(14 \%) \\
\text { aphakia; } 12 \\
(21 \%) \\
\text { complicated } \\
\text { previous }\end{array}$ & $\begin{array}{l}7(47 \%) \\
\text { aphakia; } 2 \\
(13 \%) \\
\text { complicated } \\
\text { previous }\end{array}$ & $\begin{array}{l}1(5 \%) \\
\text { aphakia; } 5 \\
(25 \%) \\
\text { complicated } \\
\text { previous }\end{array}$ & $\begin{array}{l}5(23 \%) \\
\text { complicated } \\
\text { previous } \\
\text { surgery; } 1 \\
(5 \%)\end{array}$ \\
\hline
\end{tabular}




\begin{tabular}{|c|c|c|c|}
\hline $\begin{array}{l}\text { surgery; } 3(5 \%) \\
\text { congenital } \\
\text { aniridia; } 7 \\
\text { (12\%) IOL } \\
\text { dislocation; } 1 \\
\text { (2\%) Marfan's; } 1 \\
(2 \%) \text { PDS; } 14 \\
(25 \%) \text { PEX; } 1 \\
(2 \%) \text { TASS; } 8\end{array}$ & $\begin{array}{l}\text { surgery; } 1 \\
(7 \%) \\
\text { congenital } \\
\text { aniridia; } 2 \\
\text { (13\%) IOL } \\
\text { dislocation; } \\
2(13 \%) \text { PEX; } \\
1(7 \%) \\
\text { trauma }\end{array}$ & $\begin{array}{l}\text { surgery; } 1 \text { (5\%) } \\
\text { congenital } \\
\text { aniridia; } 1 \\
\text { (5\%) IOL } \\
\text { dislocation; } 1 \\
\text { (5\%) Marfan's; } \\
6(30 \%) \text { PEX; } 4 \\
(20 \%) \text { trauma; } \\
1(5 \%) \text { uveitis }\end{array}$ & $\begin{array}{l}\text { congenital } \\
\text { aniridia; } 4 \\
\text { (18\%) IOL } \\
\text { dislocation; } 1 \\
\text { (5\%) PDS; } 6 \\
27 \%) \text { PEX; } 1 \\
(5 \%) \text { TASS; } 3 \\
14 \%) \text { trauma; } \\
1(5 \%) \text { uveitis }\end{array}$ \\
\hline
\end{tabular}

(14\%) trauma; 2

(4\%) uveitis

Sub-group

comparison:

Kruskall-

Wallis test

(asymptotic

significance

(p); $\alpha=0,05)$ :

$\begin{array}{ll}\text { Age: } p=0.323 & \text { SE: } p= \\ & 0.812\end{array}$

SE: $p=$
0.812

$A L: p=0.09$

ACD: $p=$
0.570

TK1: TK2:

$\mathrm{p}=\quad \mathrm{p}=$

$0.417 \quad 0.325$ 


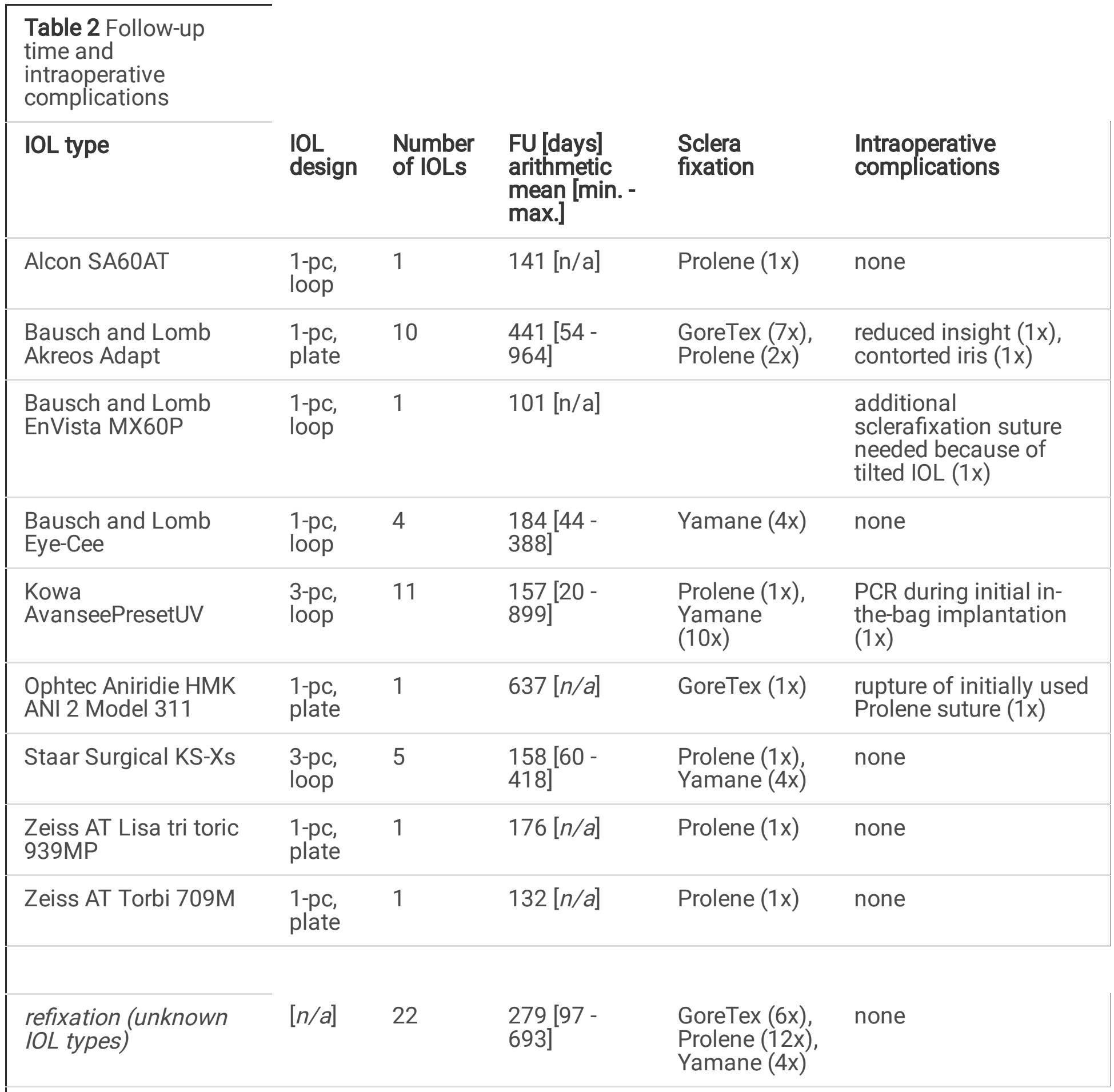

All sclera fixation techniques showed a good safety profile (Table 3). The reviewed datasets did not show a single case of retinal detachment. Intraocular pressure (IOP), central macular thickness (CMT) and central corneal thickness (CCT) did not show statistically significant differences pre-surgery compared to postsurgery (Table 3$)$. When analyzing the subgroups, the GoreTex ${ }^{\circledR}$ showed 2 (13.3\%) eyes with IOL dislocation that needed refixation. The Prolene ${ }^{\circledR}$ group included $3(15.8 \%)$ eyes that needed refixation due to IOL dislocation. One (4.5\%) eye in the sutureless flanged group needed IOL refixation. In total, 6 (10.5\%) of the eyes needed a revision of the IOL fixation or an IOL exchange. Mean surgery time was fastest for the sutureless flanged group, being approximately 1.5 times faster than GoreTex ${ }^{\circledR}$ and 2.0 times faster than 
Prolene ${ }^{\circledR}$ fixation (Table 3). Analyzing the surgery times of aphakic patients only shows the same results (Table 3).

Regarding the whole patient group $(n=57)$ a statistically significant improvement of the best-corrected visual acuity (BCVA) post-sclera fixation could be noted using a Wilcoxon signed rank test $(\mathrm{z})$ with 2-tailed significance (p): $z=-3.222$ ( $p<0.001)$. Pearson's effect size $(r)$ was calculated using the following formula: see formula 2 in the supplementary files.

Classification of the effect size was done according to Cohen $^{17}$ : $0.1<=r<0.3$ : weak; $0.3<=r<0.5$ : medium; $r$ $>0.5$ : strong.

A weak effect for the BCVA difference was calculated ( $r=0.293)$. Analyzing the subgroups only the largest group, the sutureless flanged group $(n=22)$, could present with a statistically significant BCVA difference per/post-surgery: $z=-1.988(p=0.047)$ with a medium effect size $(r=0.300)$ (Table 3). Neither the GoreTex ${ }^{\circledR}$ nor the Prolene ${ }^{\circledR}$ group showed significant differences between pre- and post-surgery. The BCVA of the three subgroups was homogenous as they did neither differ pre-surgery $(p=0.815)$ nor post-surgery $(p=0.286)$ (Table 3).

Calculated power of the Wilcoxon signed rank test of the best-corrected visual acuity of the total cohort was 0.95 . 


\begin{tabular}{|llllllllll}
$\begin{array}{l}\text { Table } 3 \\
\text { Safety and } \\
\text { efficacy } \\
\text { profile }\end{array}$ & $\begin{array}{l}\text { Total } \\
\text { patients }\end{array}$ & & z & GoreTex ${ }^{\circledR}$ & $z$ & Prolene $^{\circledR}$ & $z$ & Yamane & z \\
\end{tabular}

\section{Safety $>=\mathbf{2 0}$ days post-surgery}

\begin{tabular}{|c|c|c|c|c|c|c|c|c|}
\hline Eyes w/ RD & 0 & & 0 & & 0 & & 0 & \\
\hline $\begin{array}{l}\text { Eyes } w / I O L \\
\text { refixation }\end{array}$ & $\begin{array}{l}6 \\
(10.5 \%)\end{array}$ & & $2(13.3 \%)$ & & $\begin{array}{l}3 \\
(15.8 \%)\end{array}$ & & $1(4.5 \%)$ & \\
\hline \multirow{2}{*}{$\begin{array}{l}\text { IOP pre } \\
{[\mathrm{mmHg}]} \\
\text { mean } \pm \text { SD } \\
{[95 \% \mathrm{Cl}]}\end{array}$} & $\begin{array}{l}17.3 \pm \\
7.7\end{array}$ & $\begin{array}{l}z= \\
-1.319\end{array}$ & $\begin{array}{l}15.8 \pm \\
6.6\end{array}$ & $\begin{array}{l}z= \\
-0.157\end{array}$ & $\begin{array}{l}17.8 \pm \\
10.5\end{array}$ & $\begin{array}{l}z= \\
-0.747\end{array}$ & $\begin{array}{l}18.0 \pm \\
5.1\end{array}$ & $\begin{array}{l}z= \\
-1.308\end{array}$ \\
\hline & $\begin{array}{l}\text { [15.3; } \\
19.3]\end{array}$ & $\begin{array}{l}p= \\
0.187\end{array}$ & $\begin{array}{l}{[12.2 ;} \\
19.4]\end{array}$ & $\begin{array}{l}\mathrm{p}= \\
0.875\end{array}$ & $\begin{array}{l}{[12.8 ;} \\
22.7]\end{array}$ & $\begin{array}{l}\mathrm{p}= \\
0.455\end{array}$ & $\begin{array}{l}{[15.7 ;} \\
20.2]\end{array}$ & \multirow{3}{*}{$\begin{array}{l}\mathrm{p}=0.191 \\
\mathrm{n}=22\end{array}$} \\
\hline \multirow{2}{*}{$\begin{array}{l}\text { IOP post } \\
{[\mathrm{mmHg}]} \\
\text { mean } \pm \text { SD } \\
{[95 \% \mathrm{Cl}]}\end{array}$} & $\begin{array}{l}16.0 \pm \\
5.2\end{array}$ & $\mathrm{n}=57$ & $\begin{array}{l}16.3 \pm \\
3.9\end{array}$ & $n=15$ & $\begin{array}{l}15.6 \pm \\
5.6\end{array}$ & $n=20$ & $\begin{array}{l}16.4 \pm \\
5.6\end{array}$ & \\
\hline & $\begin{array}{l}\text { [14.6; } \\
17.4]\end{array}$ & & $\begin{array}{l}\text { [13.8; } \\
18.2]\end{array}$ & & $\begin{array}{l}\text { [12.9; } \\
18.3]\end{array}$ & & $\begin{array}{l}\text { [13.9; } \\
19.0]\end{array}$ & \\
\hline \multirow{2}{*}{$\begin{array}{l}\text { CMT pre } \\
\text { [jm] mean } \\
\pm \text { SD }[95 \% \\
\text { Cl] }\end{array}$} & $\begin{array}{l}326.0 \pm \\
93.4\end{array}$ & $\begin{array}{l}z= \\
-0.414\end{array}$ & $\begin{array}{l}415.8 \pm \\
161.6\end{array}$ & $\begin{array}{l}z= \\
-1.604\end{array}$ & $\begin{array}{l}308.8 \pm \\
83.7\end{array}$ & $\begin{array}{l}z= \\
-0.0105\end{array}$ & $\begin{array}{l}303.9 \pm \\
42.5\end{array}$ & $\begin{array}{l}z= \\
-0.415\end{array}$ \\
\hline & $\begin{array}{l}{[284.6 ;} \\
367.4]\end{array}$ & $\begin{array}{l}p= \\
0.679\end{array}$ & $\begin{array}{l}\text { [158.6; } \\
672.9]\end{array}$ & $\begin{array}{l}p= \\
0.109\end{array}$ & $\begin{array}{l}{[238.8 ;} \\
378.7]\end{array}$ & $\begin{array}{l}p= \\
0.917\end{array}$ & $\begin{array}{l}{[273.5 ;} \\
334.3]\end{array}$ & \multirow{3}{*}{$\begin{array}{l}\mathrm{p}=0.678 \\
\mathrm{n}=9\end{array}$} \\
\hline \multirow{2}{*}{$\begin{array}{l}\text { CMT post } \\
\text { [jm] mean } \\
\pm \text { SD }[95 \% \\
\text { Cl] }\end{array}$} & $\begin{array}{l}322.5 \pm \\
98.9\end{array}$ & $\mathrm{n}=18$ & $\begin{array}{l}303.6 \pm \\
87.2\end{array}$ & $\mathrm{n}=3$ & $\begin{array}{l}371.0 \pm \\
165.2\end{array}$ & $n=6$ & $\begin{array}{l}309.0 \pm \\
49.0\end{array}$ & \\
\hline & $\begin{array}{l}{[287.5 ;} \\
357.6]\end{array}$ & & $\begin{array}{l}\text { [236.5; } \\
370.6]\end{array}$ & & $\begin{array}{l}{[232.9 ;} \\
509.1]\end{array}$ & & $\begin{array}{l}\text { [282.9; } \\
335.1]\end{array}$ & \\
\hline \multirow{2}{*}{$\begin{array}{l}\text { CCT pre } \\
{[\mu \mathrm{m}] \text { mean }} \\
\pm \text { SD }[95 \% \\
\text { Cl] }\end{array}$} & \multirow{2}{*}{$\begin{array}{l}606.5 \pm \\
119.6 \\
\text { [568.7; } \\
644.2]\end{array}$} & $\begin{array}{l}z= \\
-0.140\end{array}$ & $\begin{array}{l}635.5 \pm \\
70.0\end{array}$ & $\begin{array}{l}z= \\
-0.761\end{array}$ & $\begin{array}{l}594.7 \pm \\
91.7\end{array}$ & $\begin{array}{l}z= \\
-1.268\end{array}$ & $\begin{array}{l}597.0 \pm \\
167.4\end{array}$ & \multirow{4}{*}{$\begin{array}{l}z= \\
-0.784 ; \\
p=0.433 ; \\
n=12\end{array}$} \\
\hline & & $\begin{array}{l}p= \\
0.889\end{array}$ & $\begin{array}{l}\text { [588.4; } \\
682.5]\end{array}$ & $\begin{array}{l}p= \\
0.447\end{array}$ & $\begin{array}{l}{[543.9 ;} \\
645.4]\end{array}$ & $\begin{array}{l}p= \\
0.205 ;\end{array}$ & $\begin{array}{l}{[504.3 ;} \\
689.7]\end{array}$ & \\
\hline \multirow{2}{*}{$\begin{array}{l}\text { CCT post } \\
{[\mu \mathrm{m}] \text { mean }} \\
\pm \text { SD }[95 \% \\
\text { Cl] }\end{array}$} & $\begin{array}{l}584.0 \pm \\
64.9\end{array}$ & $n=26$ & $\begin{array}{l}590.3 \pm \\
60.4\end{array}$ & $\mathrm{n}=7$ & $\begin{array}{l}601.6 \pm \\
77.3\end{array}$ & $\mathrm{n}=7$ & $\begin{array}{l}570.2 \pm \\
61.4\end{array}$ & \\
\hline & $\begin{array}{l}{[561.7 ;} \\
606.3]\end{array}$ & & $\begin{array}{l}{[547.1-} \\
633.5]\end{array}$ & & $\begin{array}{l}{[542.2 ;} \\
661.0]\end{array}$ & & $\begin{array}{l}\text { [537.5; } \\
602.9]\end{array}$ & \\
\hline
\end{tabular}

$\begin{array}{llll}\text { Subgroup } & \text { IOPpre: } p=0.232 / & \text { CMTpre: } p=0.201 / \text { CMTpost: } p & \text { CCTpre: } p=0.083 / \\ \text { comparison } & \text { IOPpost: } p=0.082 & =0.222 & \text { CCTpost: } p=0.296\end{array}$ $\mathrm{H}$

\section{Efficacy $>=20$ days post-surgery}

\begin{tabular}{|c|c|c|c|c|c|c|c|c|}
\hline \multirow{2}{*}{$\begin{array}{l}\text { BCVA pre } \\
\text { [logMAR] } \pm \\
\text { SD }[95 \% \text { Cl] }\end{array}$} & $\begin{array}{l}0.964 \pm \\
0.753\end{array}$ & $\begin{array}{l}z= \\
-3.133\end{array}$ & $\begin{array}{l}0.832 \pm \\
0.553\end{array}$ & $\begin{array}{l}z= \\
-1.398\end{array}$ & $\begin{array}{l}1.058 \pm \\
0.817\end{array}$ & $\begin{array}{l}z= \\
-1.887\end{array}$ & $\begin{array}{l}0.969 \pm \\
0.827\end{array}$ & $\begin{array}{l}z= \\
-1.988\end{array}$ \\
\hline & $\begin{array}{l}{[0.764 ;} \\
1.164]\end{array}$ & $\begin{array}{l}p= \\
0.002\end{array}$ & $\begin{array}{l}{[0.526 ;} \\
1.138]\end{array}$ & $\begin{array}{l}p= \\
0.162\end{array}$ & $\begin{array}{l}{[0.676 ;} \\
1.440]\end{array}$ & $\begin{array}{l}\mathrm{p}= \\
0.059\end{array}$ & $\begin{array}{l}{[0.602 ;} \\
1.336]\end{array}$ & \multirow{2}{*}{$\begin{array}{l}\mathrm{p}=0.047 \\
\mathrm{n}=22\end{array}$} \\
\hline & & $\mathrm{n}=57$ & & $\mathrm{n}=15$ & & $\mathrm{n}=20$ & & \\
\hline
\end{tabular}




\begin{tabular}{|c|c|c|c|c|c|}
\hline \multirow{2}{*}{$\begin{array}{l}\text { BCVA post } \\
{[\text { logMAR] } \pm} \\
\text { SD }[95 \% \mathrm{Cl}]\end{array}$} & $\begin{array}{l}0.654 \pm \\
0.612\end{array}$ & \multirow{2}{*}{$\begin{array}{l}r= \\
0.293 \\
\text { (weak) }\end{array}$} & \multirow{2}{*}{$\begin{array}{l}0.659 \pm \\
0.619 \\
{[0.316 ;} \\
1.001]\end{array}$} & \multirow{2}{*}{$\begin{array}{l}0.752 \pm \\
0.571 \\
{[0.485 ;} \\
1.020]\end{array}$} & \multirow{2}{*}{$\begin{array}{l}0.562 \pm \\
0.657 \\
{[0.271} \\
- \\
0.853]\end{array}$} \\
\hline & $\begin{array}{l}{[0.492} \\
0.817]\end{array}$ & & & & \\
\hline $\begin{array}{l}\text { SE pre }[D] \\
\text { mean } \pm \text { SD } \\
{[95 \% \mathrm{Cl}]}\end{array}$ & \multicolumn{2}{|c|}{$\begin{array}{l}4.074 \pm 7.487 \\
{[1.741 ; 6.408]}\end{array}$} & $\begin{array}{l}6.170 \pm 7.759 \\
{[0.958 ; 11.383]}\end{array}$ & $\begin{array}{l}2.018 \pm 7.884 \\
{[-2.534 ; 6.570]}\end{array}$ & $\begin{array}{l}4.412 \pm 6.977[0.825 ; \\
7.999]\end{array}$ \\
\hline $\begin{array}{l}\text { SE post [D] } \\
\text { mean } \pm \text { SD } \\
{[95 \% \mathrm{Cl}]}\end{array}$ & \multicolumn{2}{|c|}{$\begin{array}{l}-1.227 \pm 2.047 \\
{[-1.857 ;-0.597]}\end{array}$} & $\begin{array}{l}-1.563 \pm 1.391 \\
{[-2.447 ;-0.678]}\end{array}$ & $\begin{array}{l}-1.308 \pm 2.426 \\
{[-2.774 ; 0.158]}\end{array}$ & $\begin{array}{l}-0.944 \pm 2.185 \\
{[-2.031 ; 0.142]}\end{array}$ \\
\hline \multirow{2}{*}{$\begin{array}{l}\text { Surgery } \\
\text { time } \\
\text { [minutes] } \\
\text { mean } \pm \text { SD } \\
{[95 \% \mathrm{Cl}]}\end{array}$} & & & $\begin{array}{l}73.6 \pm \\
34.3\end{array}$ & $\begin{array}{l}80.8 \pm \\
28.6\end{array}$ & $\begin{array}{l}51.3 \pm \\
23.5\end{array}$ \\
\hline & & & $\begin{array}{l}{[49.1 ;} \\
98.1]\end{array}$ & $\begin{array}{l}{[58.8 ;} \\
102.8]\end{array}$ & $\begin{array}{l}{[36.4 ;} \\
66.3]\end{array}$ \\
\hline \multirow{2}{*}{$\begin{array}{l}\text { Surgery } \\
\text { time } \\
\text { [minutes] } \\
\text { aphakic } \\
\text { mean } \pm \text { SD } \\
{[95 \% \text { Cl] }}\end{array}$} & & & $\begin{array}{l}76.9 \pm \\
37.4\end{array}$ & $\begin{array}{l}98.6 \pm \\
23.8\end{array}$ & \multirow{2}{*}{$\begin{array}{l}35.6 \pm \\
14.0 \\
{[18.2} \\
53.0]\end{array}$} \\
\hline & & & $\begin{array}{l}{[45.6 ;} \\
108.2]\end{array}$ & $\begin{array}{l}\text { [69.1; } \\
128.1]\end{array}$ & \\
\hline $\begin{array}{l}\text { Subgroup } \\
\text { comparison } \\
\text { H }\end{array}$ & \multicolumn{2}{|c|}{$\begin{array}{l}\text { BCVA pre: } p=0.815 / \\
\text { BCVA post: } p=0.286\end{array}$} & Surgery & $p=0.042$ & $\begin{array}{l}\text { Surgery time aphakic } \\
\text { patients } p=0.007\end{array}$ \\
\hline
\end{tabular}

\section{Discussion}

Having been first published in 1986 by Malbran et al. ${ }^{21}$ sclera fixation of IOLs often is the only option for visual rehabilitation in complex cases. ${ }^{22}$ Furthermore, with pseudophakic ageing and an epidemiologic shift towards pseudophakia the number of IOLs needing revision or exchange is increasing. Therefore, the evaluation of IOL refixation and exchange methods gains relevance. Vounotrypidis et al. compared all secondary IOL implantation techniques but they did not differentiate which sclera fixation technique was used. ${ }^{23}$ No statistically significant difference between all secondary IOL positions could be shown. ${ }^{23}$

As to our best knowledge this is the first study that directly compares the currently most commonly used sclera fixation techniques: GoreTex ${ }^{\circledR}$ suture, Prolene ${ }^{\circledR}$ suture, sutureless flanged intrascleral IOL fixation double-needle technique. All techniques in our study showed a good safety profile without complications neither in the anterior nor in the posterior segment. Revision rates in patients with symptomatic visual 
impairment due to re-decentration or re-dislocation were generally medium and varied depending on the technique used. Amongst the subgroups the sutureless flanged technique showed the lowest IOL refixation rates after three months of follow-up. This is striking as the sutureless sclera fixation is expected to be prone to IOL tilt. ${ }^{24}$ Long-term stability and complications profile of the suture material was found to be better when thicker sutures were used. That is the reason why many surgeons switched from Prolene ${ }^{\circledR} 10-0$ to $9-0$ and started using expanded polytetrafluoroethylene (ePTFE) monofilament GoreTex CV-8 ${ }^{\circledR}$ (thickness equals Prolene $\left.{ }^{\circledR} 7-0\right) .{ }^{25,26}$ The absence of statistically significant of pre-surgery compared to post-surgery changes in IOP, CCT, CMT lets draw the conclusion that the evaluated IOL sclera fixation techniques seem to have a low surgery-associated complication rate. What concerns the corneal status, pre- and postoperative endothelial cell count would have been more accurate to evaluate corneal safety of each surgical technique as it eliminates bias by epithelial swelling. However, endothelial cell count is not a standard measurement for IOL patients in our hospital. Therefore, we could only rely on CCT that is assessed in every patient by Oculus Pentacam ${ }^{\circledR}$ and Zeiss IOL Master $700^{\circledR}$.

Efficacy evaluation of our data set has to be done very carefully. A considerable number of the included patients had reported to our hospital for the first time at the time of the IOL complication and had initially been operated elsewhere. Consequently, we had restricted access to BCVA and manifest refraction data before the IOL complication occurred. This made efficacy evaluation between pre- and post-IOL sclera fixation a challenge and represents one of the flaws of this study. However, analyzing the available data statistically significant BCVA improvement could be found for the whole patient group and for the sutureless flanged group. In the GoreTex ${ }^{\circledR}$ and Prolene ${ }^{\circledR}$ group BCVA pre-surgery did not show a statistically significant change compared to BCVA post-surgery. One might be able to conclude that a visual rehabilitation to the status pre-surgery is possible. More patients and more data are necessary for each subgroup to get more reliable statistical results. Besides the small cohort size another flaw of this study is its retrospective design.

With this study we were able to show that GoreTex ${ }^{\circledR}$ and Prolene ${ }^{\circledR}$ showed an equally good safety and efficacy profile. In our dataset the sutureless flanged group showed lower refixation rates and a good postoperative BCVA improvement. This effect might be due to the fact that the sutureless flanged was the largest subgroup. However, no conclusion about the superiority of one IOL sclera fixation technique should be drawn. Results for the patient are more likely to be dependent on the surgeon's learning curve. Currently, there might be a shift towards the sutureless sclera fixation techniques as they are fast and not prone to suture complications such as suture degradation with risk for suture breaks, suture exposure with irritation and inflammation of the surrounding tissue. ${ }^{27}$ However, the sutureless flanged technique requires three-piece IOLs with thin haptics and does not work with single-piece IOLs. As different IOL types obviously require different techniques it therefore will be wise for the surgeon to have at least one sutured sclera fixation technique in his repertoire. This study shows that all three techniques are safe techniques with low procedure-associated complication rates. However, compared to iris fixated IOLs, sclera fixation is surgically more complex and shows a steeper learning curve for the surgeon.

\section{References}


1 Foroozan, R., Tabas, J. G. \& Moster, M. L. Recurrent microhyphema despite intracapsular fixation of a posterior chamber intraocular lens. J Cataract Refract Surg29, 1632-1635, doi:10.1016/s08863350(03)00122-6 (2003).

2 Chang, D. F. et al. Complications of sulcus placement of single-piece acrylic intraocular lenses: recommendations for backup IOL implantation following posterior capsule rupture. J Cataract Refract Surg35, 1445-1458, doi:10.1016/j.jcrs.2009.04.027 (2009).

3 Crowell, E. L. Uveitis-Glaucoma-Hyphema Syndrome, <eyewiki.aao.org/Uveitis-GlaucomaHyphema_Syndrome> (2019).

4 Pueringer, S. L., Hodge, D. O. \& Erie, J. C. Risk of late intraocular lens dislocation after cataract surgery, 1980-2009: a population-based study. American journal of ophthalmology152, 618-623, doi:10.1016/j.ajo.2011.03.009 (2011).

5 Kanski, J. J. \& Bowling, B. Kanski's Clinical Ophthalmology. 8th edition edn, 928 (W.B. Saunders Ldt. (Elsevier), 2015).

6 Raghunath, M., Nienaber, C. \& Kodolitsch, Y. v. 100 Jahre Marfan-Syndrom -eine Bestandsaufnahme. Dtsch Arztebl Internationa/94, 821- (1997).

7 Steinmann, B. in Das Ehlers-Danlos-Syndrom : eine interdisziplinäre Herausforderung (ed Andreas Luttkus) Ch. 1, 1-11 (Walter de Gruyter GmbH \& Co. KG, 2011).

8 Tsilou, E. \& MacDonald, I. M. in GeneReviews((R)) (eds M. P. Adam et al.) (1993).

9 Morris, A. A. et al. Guidelines for the diagnosis and management of cystathionine beta-synthase deficiency. J Inherit Metab Dis40, 49-74, doi:10.1007/s10545-016-9979-0 (2017).

10 Sacharow, S. J., Picker, J. D. \& Levy, H. L. in GeneReviews((R)) (eds M. P. Adam et al.) (1993).

11 Fernandez-Buenaga, R. et al. Late in-the-bag intraocular lens dislocation requiring explantation: risk factors and outcomes. Eye (Lond)27, 795-801; quiz 802, doi:10.1038/eye.2013.95 (2013).

12 Hoffman, R. S., Fine, I. H. \& Packer, M. Scleral fixation without conjunctival dissection. J Cataract Refract Surg32, 1907-1912, doi:10.1016/j.jcrs.2006.05.029 (2006).

13 Yamane, S., Sato, S., Maruyama-Inoue, M. \& Kadonosono, K. Flanged Intrascleral Intraocular Lens Fixation with Double-Needle Technique. Ophthalmology124, 1136-1142, doi:10.1016/j.ophtha.2017.03.036 (2017).

14 Lewis, J. S. Ab externo sulcus fixation. Ophthalmic Surg22, 692-695 (1991).

15 Hoffman, R. S., Fine, I. H., Packer, M. \& Rozenberg, I. Scleral fixation using suture retrieval through a scleral tunnel. J Cataract Refract Surg32, 1259-1263, doi:10.1016/j.jcrs.2006.02.065 (2006). 
16 Field, A. Discovering Statistics Using SPSS. 3rd edn, (SAGE Publications Ltd., 2009).

17 Cohen, J. Statistical Power Analysis for the Behavioral Sciences 2nd Edition. 2 edn, (Lawrence Erlbaum Associates, 1988).

18 Faul, F., Erdfelder, E., Lang, A.-G. \& Buchner, A. G*Power 3.1 - Statistical Power Analyses, $<$ https://www.psychologie.hhu.de/arbeitsgruppen/allgemeine-psychologie-undarbeitspsychologie/gpower.html> (2009).

19 Faul, F., Erdfelder, E., Buchner, A. \& Lang, A. G. Statistical power analyses using G*Power 3.1: tests for correlation and regression analyses. Behavior research methods41, 1149-1160, doi:10.3758/BRM.41.4.1149 (2009).

20 Faul, F., Erdfelder, E., Lang, A. G. \& Buchner, A. G*Power 3: a flexible statistical power analysis program for the social, behavioral, and biomedical sciences. Behavior research methods39, 175-191, doi:10.3758/bf03193146 (2007).

21 Malbran, E. S., Malbran, E., Jr. \& Negri, I. Lens guide suture for transport and fixation in secondary IOL implantation after intracapsular extraction. Int Ophthalmo/9, 151-160, doi:10.1007/bf00159844 (1986).

22 Stem, M. S., Todorich, B., Woodward, M. A., Hsu, J. \& Wolfe, J. D. Scleral-Fixated Intraocular Lenses: Past and Present. J Vitreoretin Dis1, 144-152, doi:10.1177/2474126417690650 (2017).

23 Vounotrypidis, E. et al. Secondary intraocular lens implantation: a large retrospective analysis. Graefes Arch Clin Exp Ophthalmo/257, 125-134, doi:10.1007/s00417-018-4178-3 (2019).

24 Gelman, R. A. \& Garg, S. Novel yamane technique modification for haptic exposure after glued intrascleral haptic fixation. Am J Ophthalmol Case Rep14, 101-104, doi:10.1016/j.ajoc.2019.03.009 (2019).

25 Khan, M. A. et al. Scleral fixation of intraocular lenses using Gore-Tex suture: clinical outcomes and safety profile. The British journal of ophthalmology100, 638-643, doi:10.1136/bjophthalmol-2015-306839 (2016).

26 Price, M. O., Price, F. W., Jr., Werner, L., Berlie, C. \& Mamalis, N. Late dislocation of scleral-sutured posterior chamber intraocular lenses. J Cataract Refract Surg31, 1320-1326, doi:10.1016/j.jcrs.2004.12.060 (2005).

27 Ganekal, S., Venkataratnam, S., Dorairaj, S. \& Jhanji, V. Comparative evaluation of suture-assisted and fibrin glue-assisted scleral fixated intraocular lens implantation. J Refract Surg28, 249-252, doi:10.3928/1081597X-20120221-01 (2012).

\section{Supplementary Files}

This is a list of supplementary files associated with this preprint. Click to download.

- Tab2Followupcomplications20210429.docx 
- Tab3SafetyEfficacy20210429.docx

- Tab1Demographics20210429.docx

- formula.docx 